\title{
Tree Root
}

National Cancer Institute

\section{Source}

National Cancer Institute. Tree Root. NCI Thesaurus. Code C45800.

Concepts in a hierarchy are often said to be in a tree. A root refers to a concept that is considered as the top node for a specific purpose. 